[2] Brody GS et al (2015) Anaplastic large cell lymphoma occurring in women with breast implants: analysis of 173 cases. Plast Reconstr Surg 135(3):695-705

[3] FDA (2011) Reports of Anaplastic Large Cell Lymphoma (ALCL) in Women with Breast Implants: FDA Safety Communication http://www.fda.gov/MedicalDevices/ Safety/AlertsandNotices/ucm240000.htm

[4] BfArM (2011/2015) Möglicher Zusammenhang zwischen Brustimplantaten und der Entstehung eines anaplastischen großzelligen Lymphoms (ALCL) http://www. bfarm.de/SharedDocs/Risikoinformationen/Medizinprodukte/DE/
Brustimplantate_ALCL_FDA.html

[5] Miranda RN et al (2014) Breast implant-associated anaplastic large-cell lymphoma: Long-term follow-up of 60 patients. J Clin Oncol 32:114-120

[6] YeX et al (2014) Anaplastic large cell lymphoma (alcl) and breast implants: Breaking down the evidence. Mutat Res Rev Mutat Res 762:123-132

[7] Kim Bet al. (2015). Breast Implant-associated Anaplastic Large Cell Lymphoma: Updated Results from a Structured Expert Consultation Process. Plast Reconstr Surg Glob Open 3(1):e296

\title{
Desensibilisierungsbehandlung nach Krebs ohne Risiko möglich?
}

\section{Hintergrund}

Die spezifische Desensibilisierung ist die zurzeit einzige kausale Therapie IgE-vermittelter allergischer Erkrankungen. Im Zusammenhang mit einer Desensibilisierungstherapie bei Krebspatienten sind zwei Fragen relevant: Ist die Behandlung erfolgreich durchführbar und ist sie onkologisch sicher? Dabei ist insbesondere zu bedenken, dass das Ziel einer Desensibilisierung die Erzeugung einer verstärkten Immuntoleranz ist. Viele der immunologischen Vorgänge, die an diesem Prozess beteiligt sind, spielen allerdings auch eine Rolle bei der Krebsbekämpfung, z. B. die CTLA-4- und PD-1-abhängigen Immuncheckpoints [1]. Eine Aktivierung solcher „Bremsen“ im Immunsystem ist für die Desensibilisierung erwünscht, könnte dagegen für die Kontrolle von Krebs durch das Immunsystem ungünstig sein.

Zum Einfluss einer Desensibilisierung (Hyposensibilisierung, auch als allergenspezifische Immuntherapie oder SIT bezeichnet) auf den Verlauf oder das Rezidivrisiko bei bestehender oder durchgemachter Krebserkrankung gibt es allerdings nur wenige Daten. In einer publizierten Fallserie von 6 Patienten, die vor oder unter der SIT an Krebs erkrankten, kam es zu keinem Rückfall, der auf die Hyposensibilisierungsbehandlung zurückgeführt werden konnte. Auch in den Fällen mit während der SIT diagnostiziertem, aber frühem Tumor wurde die Desensibilisierung fortgeführt und abgeschlossen, sofern eine lebensbedrohliche Allergie bestand - ohne negative Folgen mit Blick auf die Krebserkrankung [2]. Die Autoren folgern, dass Krebs - zumindest in frühen Stadien - keine Kontraindikation für eine Desensibilisierung darstellt.

Die etwas anders gelagerte Frage, ob eine SIT das primäre Erkrankungsrisiko an Krebs beeinflusst, untersuchte eine im letzten Jahr publizierte Fall-Kontroll-Studie. Über 1100 Personen, die wegen einer Allergie eine Hyposensibilisierungsbehandlung erhalten hatten, wurden 20 Jahre nachbeobachtet [3]. Im Vergleich zu einer Kontrollgruppe allergischer Patienten ohne SIT waren abgesehen von einem etwas verringerten Risiko für chronische myeloische oder lymphatische Leukämie keine Unterschiede in der Krebsinzidenz zu erkennen. Allerdings traten insgesamt nur wenige Krebserkrankungen auf: mit SIT 89, ohne SIT 81 Fälle, das entspricht 7,71\% vs. 7,01\%. Die Teilnehmer waren mit im Mittel 43 Jahren bei Studienende in einem Alter, in dem Krebsdiagnosen naturgemäß noch selten sind.

\section{Aktuelle Leitlinie - Stabile Krebserkrankung keine Kontraindikation für Desensibilisierungsbehandlung} Laut der aktuellen deutsch-österreichisch-schweizerischen Leitlinie zur (allergen)spezifischen Immuntherapie [4] wird eine SIT bei Krebspatienten mit stabiler Erkrankung als grundsätzlich möglich angesehen. Diese Bewertung stützt sich im Wesentlichen auf die erwähnte kleine Fallserie [3]. „Maligne neoplastische Erkrankung mit aktuellem Krankheitswert" sind dagegen weiter als Kontraindikation geführt. Andere internationale Leitlinien zur Hyposensibilisierung berühren das Thema nicht. Für eine verlässliche Beurteilung der Sicherheit von SIT bei Krebspatienten reichen die wenigen vorliegenden Daten nicht aus. Es sind weitere Studien erforderlich.

Literatur

1. Akdis M, Akdis CA (2014) Mechanisms of allergen-specific immunotherapy: multiple suppressor factors at work in immune tolerance to allergens. J Allergy Clin Immunol 133(3):621-631

2. Wöhrl Set al. (2011) Malignancy and Specific Allergen Immunotherapy: The Results of a Case Series. Int Arch Allergy Immunol 156(3):313-319

3. Bozek A et al. (2014) The safety of specific immunotherapy for patients allergic to house-dust mites and pollen in relation to the development of neoplasia and autoimmune disease: a long-term, observational case-control study. Int Arch Allergy Immunol 163(4):307-312

4. S2k-Leitlinie zur (allergen-)spezifischen Immuntherapie bei IgE-vermittelten allergischen Erkrankungen (2014) Allergo J Int 23: 282 http://www.awmforg/uploads/tx_ szleitlinien/061-0041_S2k_SIT_2014-12.pdf

\section{Krebsinformationsangebote für Fachkreise}

Telefon: 0800-430 40 50, täglich 8-20 Uhr

E-Mail Service: Krebsinformationsdienst.med@dkfz.de

- Individuelle recherchierte Informationen auf der Basis der besten verfügbaren Evidenz

- Ausführliche Informationen zu mehr als 150 unkonventionellen Diagnose- und Behandlungsverfahren

- Umfassende Datenbank mit Adressen von Krebsberatungsstellen, niedergelassenen Psychoonkologen und weiteren Unterstützungsangeboten

Korrespondenzadresse

\section{A. Gaisser}

Krebsinformationsdienst, Deutsches Krebsforschungszentrum andrea.gaisser@dkfz-heidelberg.de

Quelle: Onkologe 2015 • 21:734-738 DOI 10.1007/s00761-015-3002-5

๑ Springer-Verlag Berlin Heidelberg 2015 